\title{
Totally Umbilical Semi-Invariant Submanifolds of a Nearly Sasakian Manifold
}

\author{
Khalid Ali Khan, Siraj-Uddin, and Nargis Jamal
}

ABSTRACT. We have studied some differential geometric aspects of semiinvariant submanifolds of a nearly Sasakian manifold, which led us to classify of totally umbilical semi-invariant submanifolds of a nearly Sasakian manifold.

\section{INTRODUCTION}

The study of semi-invariant submanifold or contact $C R$-submanifolds of almost contact metric manifold was initiated by A. Bejancu and N. Papaghiuc [1] and was followed up by several other geometers (c.f., [1, 9, 11] etc.). In particular, semi-invariant submanifolds of different classes of almost contact metric manifolds have also been studied (c.f., $[4,6]$ ). J.S. Kim et al. [6] initiated the study of semi-invariant submanifolds of nearly trans-Sasakian manifolds. They have obtained some basic results concerning the integrability of the distributions involved in this setting and their parallelism in the submanifold. In the present note, we have worked out a classification for totally umbilical semi-invariant submanifolds of a nearly Sasakian manifold.

\section{Preliminaries}

Let $\bar{M}$ be an almost contact metric manifold with almost contact metric structure $(\phi, \xi, \eta, g)$, that is $\phi$ is a $(1,1)$ tensor field, $\xi$ is a vector field, $\eta$ is a 1-form and $g$ is the compatible Riemannian metric such that

$$
\left\{\begin{array}{l}
\phi^{2} X=-X+\eta(X) \xi, \quad \eta(\xi)=1, \quad \phi(\xi)=0, \quad \eta \circ \phi=0, \\
g(\phi X, \phi Y)=g(X, Y)-\eta(X) \eta(Y), \quad \eta(X)=g(X, \xi),
\end{array}\right.
$$

for each $X, Y \in T \bar{M}$ where $T \bar{M}$ denotes the tangent bundle of $\bar{M}$.

An almost contact metric structure $(\phi, \xi, \eta, g)$ on $\bar{M}$ is a nearly Sasakian structure [3] if

$$
\left(\bar{\nabla}_{X} \phi\right) Y+\left(\bar{\nabla}_{Y} \phi\right) X=2 g(X, Y) \xi-\eta(Y) X-\eta(X) Y .
$$

2000 Mathematics Subject Classification. Primary: 53C40; 53B25.

Key words and phrases. Semi-invariant, totally umbilical, nearly Sasakian. 
An $m$-dimensional submanifold $M$ of $\bar{M}$ is said to be a semi-invariant submanifold if there exist a pair of orthogonal distributions $\left(D, D^{\perp}\right)$ satisfying the conditions

(i) $T M=D \oplus D^{\perp} \oplus\langle\xi\rangle$.

(ii) The distribution $D$ is invariant by $\phi$ i.e., $\phi D_{x}=D_{x} \forall x \in M$.

(iii) The distribution $D^{\perp}$ is anti-invariant i.e., $\phi D_{x}^{\perp} \subseteq T_{x}^{\perp} M, \forall x \in M$. where $\langle\xi\rangle$ is the distribution spanned by the structure vector field $\xi$.

Let $T M$ denote the tangent bundle on $M$. The orthogonal complement of $\phi D^{\perp}$ in the normal bundle $T^{\perp} M$ is an invariant sub bundle of $T^{\perp} M$ under $\phi$ and is denoted by $\mu$ i.e.,

$$
T^{\perp} M=\phi D^{\perp} \oplus \mu .
$$

For $U, V \in T M$ and $N \in T^{\perp} M$, the Gauss and Weingarten formulae are given by

$$
\begin{aligned}
& \bar{\nabla}_{U} V=\nabla_{U} V+h(U, V), \\
& \bar{\nabla}_{U} N=-A_{N} U+\nabla_{U}^{\perp} N,
\end{aligned}
$$

where $\nabla$ and $\nabla^{\perp}$ are symbols used for connection on $T M$ and $T^{\perp} M$ respectively. $h$ and $A_{N}$ denote the second fundamental forms related by $g(h(U, V), N)=g\left(A_{N} U, V\right)$ and $g$ denotes the Riemannian metric on $\bar{M}$ as well as on $M$.

The transforms $\phi U$ and $\phi N$ are decomposed into tangential and normal parts respectively as

$$
\begin{aligned}
\phi U & =P U+F U . \\
\phi N & =t N+f N .
\end{aligned}
$$

Note 2.1. It is easy to observe that $P U \in D, F U \in \phi D^{\perp}, t N \in D^{\perp}$ and $f N \in \mu$.

Now, denoting by $\mathcal{P}_{U} V$ and $\mathcal{Q}_{U} V$ the tangential and normal parts of $\left(\bar{\nabla}_{U} \phi\right) V$ and making use of equations $(2.3),(2.4),(3.4)$ and $(2.6)$, the following equations may easily be obtained

$$
\begin{aligned}
& \mathcal{P}_{U} V=\left(\nabla_{U} P\right) V-A_{F V} U-t h(U, V), \\
& \mathcal{Q}_{U} V=\left(\nabla_{U} F\right) V+h(U, P V)-f h(U, V),
\end{aligned}
$$

where the covariant derivatives of $P$ and $F$ are defined by

$$
\begin{aligned}
& \left(\nabla_{U} P\right) V=\nabla_{U} P V-P \nabla_{U} V, \\
& \left(\nabla_{U} F\right) V=\nabla_{U}^{\perp} F V-F \nabla_{U} V .
\end{aligned}
$$

A submanifold $M$ of an almost contact metric manifold $\bar{M}$ is said to be a totally umbilical submanifold if the second fundamental form satisfies

$$
h(U, V)=g(U, V) H,
$$

where $H$ is the mean curvature vector. 


\section{Semi-invariant Submanifolds of a NeARly SASAKian manifold}

To develop the proof of the main theorem, we start with the following preparatory results.

Proposition 3.1. Let $M$ be a semi-invariant submanifold of a nearly Sasakian manifold $\bar{M}$ with $h(X, \phi X)=0$ for each $X \in D \oplus\langle\xi\rangle$. If the invariant distribution $D \oplus\langle\xi\rangle$ is integrable, then each of its leaves is totally geodesic in $M$ as well as in $\bar{M}$.

Proof. For $X, Y \in D \oplus\langle\xi\rangle$, we have

$$
\begin{array}{r}
h(X, \phi Y)+h(\phi X, Y)= \\
=\left(\bar{\nabla}_{X} \phi\right) Y+\left(\bar{\nabla}_{Y} \phi\right) X+\phi\left(\bar{\nabla}_{X} Y+\bar{\nabla}_{Y} X\right)-\left(\nabla_{X} \phi Y+\nabla_{Y} \phi X\right) .
\end{array}
$$

Taking account of the hypothesis and the formula (2.2), the above equation takes the form

$$
\begin{array}{r}
0=2 g(X, Y) \xi-\eta(Y) X-\eta(X) Y+\phi\left(\nabla_{X} Y+\nabla_{Y} X\right)- \\
-\left(\nabla_{X} \phi Y+\nabla_{Y} \phi X\right)+2 \phi h(X, Y) .
\end{array}
$$

On equating the normal parts in the right hand side of the above equation to zero, we get

$$
2 f h(X, Y)=F\left(\nabla_{X} Y+\nabla_{Y} X\right),
$$

from which we deduce that

$$
\begin{gathered}
\nabla_{X} Y+\nabla_{Y} X \in D \oplus\langle\xi\rangle, \\
h(X, Y) \in \phi D^{\perp} .
\end{gathered}
$$

As $D \oplus\langle\xi\rangle$ is integrable, it follows from the observation (3.2) that

$$
\nabla_{X} Y \in D \oplus\langle\xi\rangle .
$$

Taking account of this fact in equation (3.1), it gives

$$
h(X, Y)=0 .
$$

The assertion is proved by virtue of (3.4) and (3.5).

The above proposition leads to the following consequence which is in itself an important result with geometric point of view.

Corollary 3.1. Let $M$ be a totally umbilical semi-invariant submanifold of a nearly Sasakian manifold $\bar{M}$. If the invariant distribution $D \oplus\langle\xi\rangle$ on $M$ is integrable, then $M$ is totally geodesic in $\bar{M}$. 
To workout the integrability condition for an anti-invariant distribution $D^{\perp} \oplus\langle\xi\rangle$, for $Z, W \in D^{\perp} \oplus\langle\xi\rangle$ and $U \in T M$, we write

$$
\begin{aligned}
2 g\left(A_{\phi Z} W, U\right) & =g(h(U, W), \phi Z)+g(h(U, W), \phi Z) \\
& =g\left(\bar{\nabla}_{W} U, \phi Z\right)+g\left(\bar{\nabla}_{U} W, \phi Z\right) \\
& =-g\left(\bar{\nabla}_{W} \phi U+\bar{\nabla}_{U} \phi W, Z\right)+g\left(\left(\bar{\nabla}_{U} \phi\right) W+\left(\bar{\nabla}_{W} \phi\right) U, Z\right) \\
& =g\left(\phi U, \bar{\nabla}_{W} Z\right)+g\left(A_{\phi W} U, Z\right) \\
& =-g\left(U, \phi \bar{\nabla}_{W} Z\right)+g\left(A_{\phi W} U, Z\right)
\end{aligned}
$$

As $U \in T M$, we obtain

$$
2 A_{\phi Z} W=A_{\phi W} Z-\phi \bar{\nabla}_{W} Z
$$

Interchange $Z$ and $W$, we get

$$
2 A_{\phi W} Z=A_{\phi Z} W-\phi \bar{\nabla}_{Z} W .
$$

On making use of equations (3.6) and (3.7), we get

$$
3\left(A_{\phi Z} W-A_{\phi W} Z\right)=\phi[Z, W]
$$

which on operating $\phi$ and using equation (2.1), it follows

$$
[Z, W]=\eta([Z, W]) \xi+3 \phi\left(A_{\phi W} Z-A_{\phi Z} W\right) .
$$

Equation (3.8) leads the following proposition.

Proposition 3.2. Let $M$ be a semi-invariant submanifold of a nearly Sasakian manifold $\bar{M}$, then the distribution $D^{\perp} \oplus\langle\xi\rangle$ is integrable if and only if

$$
A_{\phi Z} W=A_{\phi W} Z
$$

for each $Z, W \in D^{\perp} \oplus\langle\xi\rangle$.

Corollary 3.2. On a semi-invariant submanifold of a nearly Sasakian manifold the distribution $D^{\perp} \oplus\langle\xi\rangle$ is integrable.

\section{Totally UMBILICAL SEMI-INVARIANT SUbMANIFOLD OF NEARLY SASAKIAN MANIFOLDS}

Throughout this section $M$ denotes a totally umbilical semi-invariant submanifold of a nearly Sasakian manifold $\bar{M}$. For $U \in T M$, by formula (2.2),

$$
\left(\bar{\nabla}_{U} \phi\right) U=\|U\|^{2} \xi-\eta(U) U .
$$

In particular for $Z \in D^{\perp}$,

$$
\left(\bar{\nabla}_{Z} \phi\right) Z=\|Z\|^{2} \xi
$$

and therefore,

$$
\left\{\begin{array}{l}
\mathcal{P}_{Z} Z=\|Z\|^{2} \xi \\
\mathcal{Q}_{Z} Z=0 .
\end{array}\right.
$$


On applying equation (2.7) and using the fact that $P Z=0$, equation (4.3) gives,

$$
-\|Z\|^{2} \xi-g(H, F Z) Z-\|Z\|^{2} t H=P \nabla_{Z} Z .
$$

Now, in view of the observations made in Note 2.1, the above equation yields

$$
\left\{\begin{aligned}
g(H, F Z) Z+\|Z\|^{2} t H & =0 \\
\|Z\|^{2} \xi & =0 .
\end{aligned}\right.
$$

First part of equation (4.5) has solutions if either $(a) \operatorname{dim}\left(D^{\perp}\right)=1$ or $(b)$ $H \in \mu$.

On the other hand second part of equation (4.5) has solutions if $D^{\perp}=$ $\{0\}$.

Now, we are in a position to prove the following theorem.

Theorem 4.1. Let $M$ be a totally umbilical semi-invariant submanifold of a nearly Sasakian manifold $\bar{M}$. Then at least one of the following is true

(i) $M$ is anti-invariant.

(ii) $M$ is totally geodesic.

(iii) $\operatorname{dim}\left(D^{\perp}\right)=1$ and $D \oplus\langle\xi\rangle$ is not integrable.

Proof. If $D=\{0\}$, then by definition $M$ is anti-invariant which is case (i). If $D \neq\{0\}$ and $D \oplus\langle\xi\rangle$ is integrable then by Corollary 3.1, $M$ is totally geodesic in $\bar{M}$ which accounts for case (ii). If $D \oplus\langle\xi\rangle$ is not integrable and $H \in \mu$ then by virtue of observation (3.3), $M$ is again totally geodesic. If however $H \notin \mu$, then equation (4.5) has solutions if and only if dim $\left(D^{\perp}\right)=1$ or $D^{\perp}=\{0\}$. For the case when $\operatorname{dim}\left(D^{\perp}\right)=1, M$ belongs to the class (iii). Lastly, if $H \notin \mu$ and $D^{\perp}=\{0\}$, then again by Corollary 3.1, $M$ is totally geodesic. This completes the proof.

\section{REFERENCES}

[1] A. Bejancu and N. Papaghiuc, Semi-invariant submanifold of Sasakian manifold, An. St. Univ. AI.I. Cuza. Iasi, 27(1981), 163-170.

[2] D.E. Blair, Contact manifolds in Riemannian geometry, Lecture Notes in Math., Vol. 509, Springer-Verlag, 1976.

[3] D.E. Blair, D.K. Showers and K. Yano, Nearly Sasakian structures, Kodai Math. Sem. Rep., 27(1976), 175-180.

[4] C. Calin, Contact CR-Submanifolds of a nearly Sasakian manifold, Demonstratio Math., 29(1996), 337-348.

[5] C. Gherghe, Harmonicity on nearly trans-Sasaki manifolds, Demonstratio Math., 33(2000), 151-157.

[6] Jeong-Sik Kim, Ximin Liu and M.M. Tripathi, On Semi-invariant submanifolds of Nearly trans-Sasakian manifolds, Int. J. Pure and Appl. Math. Sci., 1(2004), 15-34.

[7] M. Kobayashi, CR-submanifolds of a Sasakian manifold, Tensor, 35(1981), 297-307. 
[8] M. Kobayashi, Semi-invariant submanifolds of a certain class of almost contact manifold, Tensor, 43(1986), 28-36.

[9] K. Matsumoto, M.H. Shahid, I. Mihai, Semi-invariant submanifolds of certain almost contact manifolds, Bull. Yamagata Univ. Natur. Sci., 13(1994), No. 3, 183-192.

[10] J.A. Oubina, New classes of almost contact metric structures, Publ. Math. Debrecen, 32(1985), 187-193.

[11] K. Yano and M. Kon, On contact CR-submanifolds, J. Korean Math. Soc., 26(1989), 231-262.

\author{
Khalid Ali Khan \\ Department of Mathematics \\ Aligarh Muslim University \\ AligARH-202 002 \\ INDIA \\ E-mail address: khalid.mathematics@gmail.com \\ SIRAJ-UDDIN \\ Department of Mathematics \\ Aligarh Muslim University \\ AligARH-202 002 \\ INDIA \\ E-mail address: siraj@math.com
}

